\title{
Doktor Fango mag Mango
}

Zeno Schneider

Korrespondenz:

Dr. med. Zeno Schneider

Benzigerstrasse 7

CH-8840 Einsiedeln

Tel. 0554126153
Personen

Dr. Fango,

ein älterer Internist mit latentem Hang zum

Exotischen

Fräulein Zindel,

eine hochengagierte Praxisgehilfin

Ein Karton voller reifer Mango

(erste Qualität)

Ein Tastentelefon

(Tritel Flims Swisscom, beige)

Eine Praxistür

(Eichenimitat, edel)

Eine Türklingel

(Tonlage: B-Dur)

\section{Bühnenbild}

Eine emsige Arztpraxis in einem emsigen Dorf

\section{Zeit}

Heute

Draussen blinkt die Maisonne aus den aufgerissenen Wolkenbändern. Im Hintergrund heulen leise Ambulanzsirenen. Verkehrsrauschen und vergnügliches Vogelzwitschern dringen durch die angelehnten Fenster.

Dr. Fango brütet über seinen Unterlagen.

Fräulin Zindel brütet über ihren Unterlagen.

Es gibt nichts in diesem Raum, das nicht über Unterlagen brütet.

Dr. Fango: Fräulein Zindel!

Frl. Zindel: Herr Doktor, was gibt's denn?

Dr. Fango: Geben Sie mir doch mal die KG Wagner.

Frl. Zindel: Sofort, Herr Doktor!

Hier, bitte schön. - Brauchen Sie auch die Laborblätter?

Dr. Fango: Ach, Fräulein Zindel, Sie und Ihre Laborblätter!

Frl. Zindel: Bei Herrn Doktor Fankhau ...

Dr. Fango: Ach, Fräulein Zindel, - Sie und Ihr Doktor Fankhauser!

Telefon: $\quad$ Dring-dring - dring ...
Frl. Zindel: Entschuldigen Sie, Herr Doktor, das Telefon ...

Dr. Fango: Was drängt, muss sein, Fräulein Zindel, tun Sie, was Sie können sollten.

Telefon: Ddrinnnggg-ddrinnngg!

Frl. Zindel: Praxis Dr. Fango, Zindel, was können wir für uns tun?

- Nein, der ist leider noch nicht ...

- Ja, versuchen Sie's doch in zwei, ja, genau, in zwei Tagen, aber ...

- Nein, nein, das spielt für uns keine Rolle ...

- Ja, sehr gern, vielen Dank! Auf Wiedersehen.

Dr. Fango: War das Göhler?

Frl. Zindel: Nein, Herr Doktor!

Dr. Fango: Hätte ja sein können, um diese Zeit.

Frl. Zindel: Da haben Herr Doktor recht.

Dr. Fango: Wie immer, Fräulein Zindel, wie immer. Sie finden mich im Sprechzimmer zwo, wenn Sie mich brauchen

(Ab ins Sprechzimmer zwo.)

Frl. Zindel: Bei Doktor Fankhaus ...

Dr. Fango (brüllt aus dem Zimmer zwo):

Zindelchen, lassen Sie mich mit diesem Fankhauser in Ruhe!

Frl. Zindel: Ich meine ja bloss.

Türklingel: Schrill - schrriiiilll

Praxistür: Ääch - z-tog

Mangokarton: Guten Tag, - ich soll mich hier übergeben.

Frl. Zindel: Wie bitte?

Mangokarton: Ich meine, ich soll mich hier abgeben. Frl. Zindel: Ach so, - kommen Sie doch herein!

Mangokarton: Danke! Also, ich übergebe mich jetzt.

Frl. Zindel: Darf ich Sie bitten, vorher das Personalienblatt auszufüllen? Hier, bitte schön!

Mangokarton: Ich komme aber nicht als Patient. Frl. Zindel: Das meinen hier alle.

Mangokarton: Dann bin ich eine Ausnahme.

Frl. Zindel: Das meinen alle hier. 


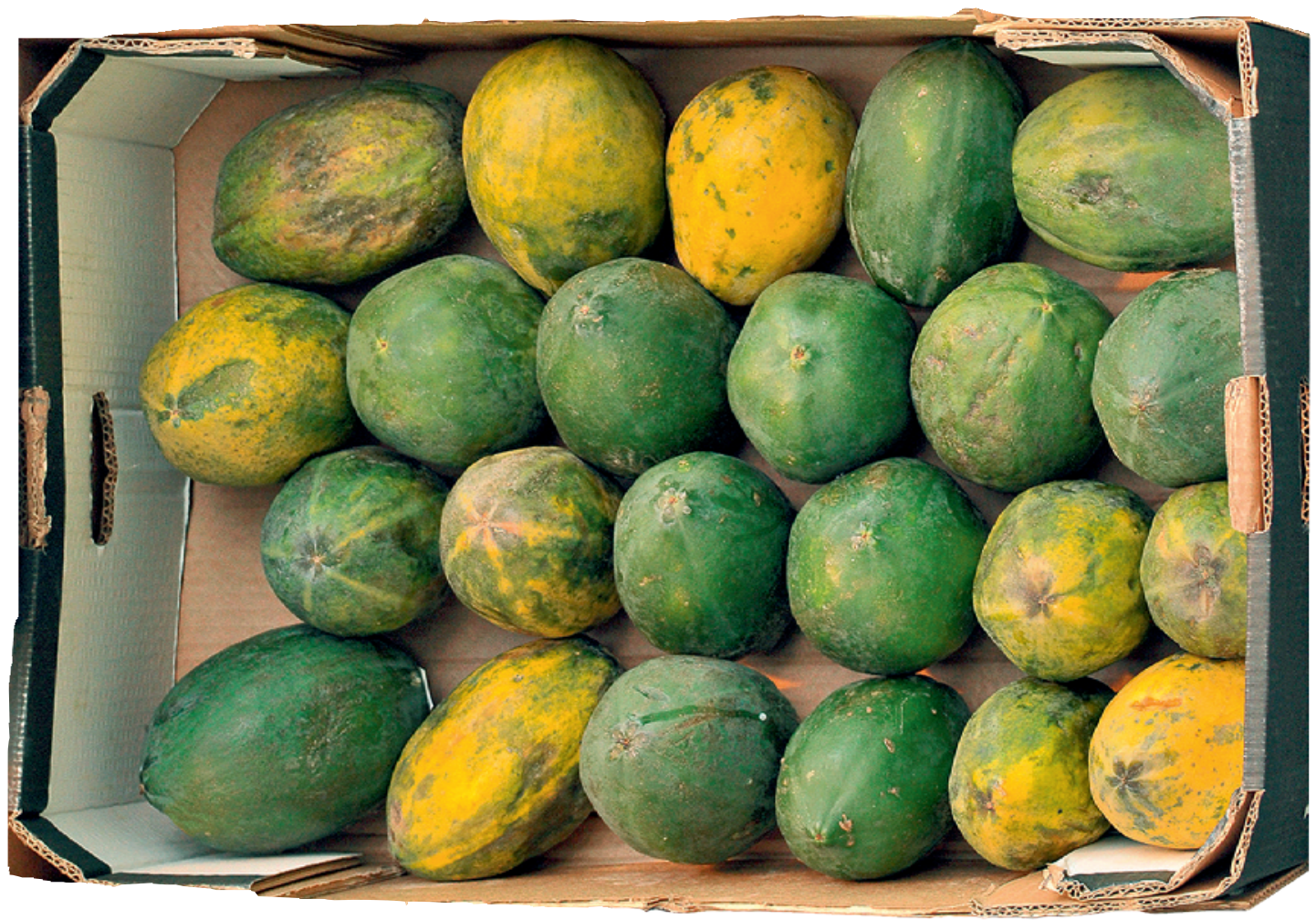

Mangokarton: Also, - nehmen Sie mich jetzt oder nicht?

Frl. Zindel: Wir nehmen keine neuen Patienten mehr. Schon seit letztem Jahr.

Mangokarton: Ich bin ja auch keiner.

Frl. Zindel: Ach so?

Mangokarton: Sagte ich das nicht schon?

Frl. Zindel: Nein, sie sagten bloss, sie kämen nicht als solcher. (Energisch) Seit ich bei Dr. Fankhau ...

Dr. Fango: (brüllt aus Zimmer zwo):

Zindel, hören Sie mir mit diesem Fankhauser auf, - aber sofort bitte!

Frl. Zindel: Verzeihen Sie, Herr Doktor, ich werde es nicht mehr tun.

Dr. Fango: Was ich sehr begrüsse, Zindelchen!

Mangokarton: Also dann gebe ich mich jetzt über.

(Legt sich verstummend auf die Empfangstheke).

Frl. Zindel: Also, bei Dr. Fankhau ...
Dr. Fango: Zindel! Jetzt reicht's aber! (Stürmt aus dem Zimmer zwo). Ja, was haben wir denn da?!

Frl. Zindel: Das kommt nicht als Patient, Herr Doktor!

Dr. Fango: Sieht aus wie ein Karton Mango! (Lechzend) M-a-a-a-ng-o-o-o!

Frl. Zindel: Da hat der Herr Doktor schon wieder recht.

Dr. Fango (appetitlich erregt) Zindelchen, Messer, bitte!

(Beide stürzen sich lüstern auf die reifen Mangos, die sie sich gierig in den Mund stopfen.)

Frl. Zindel (wischt sich den triefenden Saft vom Mund): Notfallpauschale, Herr Doktor?

Beide lachen ebenso barock wie genüsslich.

Vorhang 